\title{
Measurement of Feynman- $x$ spectra of photons and neutrons in the very forward direction in deep-inelastic scattering at HERA
}

\author{
Armen Buniatyan ${ }^{\mathrm{a}}$ on behalf of the H1 Collaboration \\ University of Birmingham
}

\begin{abstract}
Measurements of normalised cross sections for the production of photons and neutrons at very small angles with respect to the proton beam direction in deep-inelastic ep scattering at HERA are presented as a function of the Feynman variable $x_{F}$ and of the centre-of-mass energy of the virtual photon-proton system $W$. The data are taken with the H1 detector in the years 2006 and 2007 and correspond to an integrated luminosity of $131 \mathrm{pb}^{-1}$. The measurement is restricted to photons and neutrons in the pseudorapidity range $\eta>7.9$ and covers the range of negative four momentum transfer squared at the positron vertex $6<Q^{2}<100 \mathrm{GeV}^{2}$, of inelasticity $0.05<y<0.6$ and of $70<W<245 \mathrm{GeV}$. To test the Feynman scaling hypothesis the $W$ dependence of the $x_{F}$ dependent cross sections is investigated. Predictions of deep-inelastic scattering models and of models for hadronic interactions of high energy cosmic rays are compared to the measured cross sections.
\end{abstract}

\section{Introduction}

Measurements of particle production at very small polar angles with respect to the proton beam direction in positron-proton collisions are important inputs for the theoretical understanding of proton fragmentation mechanisms. Measurements of these forward particles also valuable for high energy cosmic ray experiments, as they provide important new constraints for high energy air shower models $[1,2]$.

The H1 and ZEUS experiments at the ep collider HERA have studied the production of forward baryons (protons and neutrons) and photons, which carry a large fraction of the longitudinal momentum of the incoming proton [3-9]. These analyses have demonstrated that models of deep-inelastic scattering (DIS) are able to reproduce the forward baryon measurements if contributions from different production mechanisms are considered, such as string fragmentation, pion exchange, diffractive dissociation and elastic scattering of the proton $[6,7]$. The forward photon production rate, however, is overestimated by the models by 50 to $70 \%$ [8]. The measurements also confirm the hypothesis of limiting fragmentation $[10,11]$, according to which, in the high-energy limit, the cross section for the inclusive production of particles in the target fragmentation region is independent of the incident projectile energy.

Measurements in the DIS regime provide a possibility to investigate the process at different centre-of-mass (CM) energies of the virtual photon-proton system, $W$, within the same experiment. The studies of the energy dependence of particle production allow a test of the Feynman scaling [12] hypothesis, according to which particle production is expected to show a scaling behaviour, i.e. independence of the CM energy in terms of the Feynman- $x$ variable,

a e-mail: armen.buniatyan@desy.de
$x_{F}=2 p_{\|}^{*} / W$. Here $p_{\|}^{*}$ is the longitudinal momentum of the particle in the virtual photon-proton CM frame with respect to the direction of the beam proton. In several previous measurements Feynman scaling was found to be violated in the fragmentation process in the central rapidity region: On the contrary, no sizable violation of Feynman scaling has been observed in the target fragmentation region in $p p$ and $p \bar{p}$ collisions [13]. However, these conclusions are debated [14] and the scarcity of other experimental forward particle production data motivates further studies of forward particle production.

In this report, the new $\mathrm{H} 1$ measurement of the production of neutrons and photons in DIS is presented [9]. The production is studied as a function of $x_{F}$ and $W$. This is the first direct experimental test of Feynman scaling for photons and neutrons produced in the very forward direction. Predictions from different DIS and different cosmic ray (CR) hadronic interaction Monte Carlo (MC) models are compared to the results. The simultaneous measurement of forward neutrons and photons provides a useful input for MC model development also because of the respective different production mechanisms: forward photons almost exclusively originate from decays of neutral mesons produced in the fragmentation of the proton remnant (Fig. 1a), while forward neutrons are produced also via a colour singlet exchange process (Fig. 1b).

\section{Experimental procedure}

The data used in this analysis were collected with the H1 detector at HERA in the years 2006 and 2007 and correspond to an integrated luminosity of $131 \mathrm{pb}^{-1}$. During this period HERA collided positrons and protons with energies of $E_{e}=27.6 \mathrm{GeV}$ and $E_{p}=920 \mathrm{GeV}$, respectively, corresponding to a centre-of-mass energy of $\sqrt{s}=319 \mathrm{GeV}$.

This is an Open Access article distributed under the terms of the Creative Commons Attribution License 4.0, which permits unrestricted use, distribution, and reproduction in any medium, provided the original work is properly cited. 

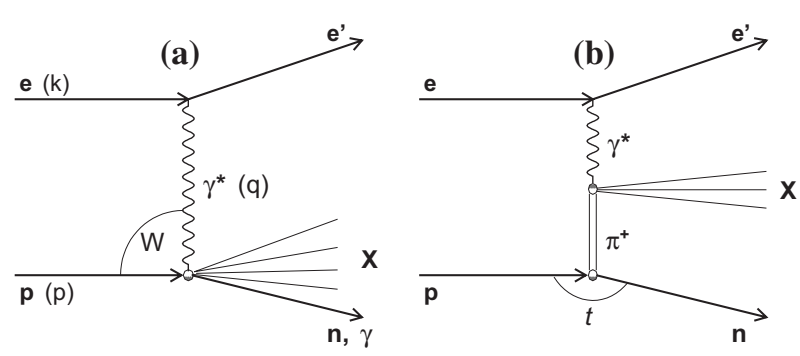

Figure 1. (a) Generic diagram for forward photon or neutron production $e p \rightarrow e^{\prime} \gamma X, e p \rightarrow e^{\prime} n X$ in deep-inelastic scattering. (b) Diagram of forward neutron production via pion exchange.

\section{1. $\mathrm{H} 1$ detector}

A detailed description of the $\mathrm{H} 1$ detector can be found elsewhere [15-18]. The interaction region is surrounded by a two-layer silicon strip detector and large concentric drift chambers, operated inside a $1.16 \mathrm{~T}$ solenoidal magnetic field. Charged particle momenta are measured in the angular range $15 \mathrm{deg}<\theta<165 \mathrm{deg}$. The forward tracking detector is used to supplement track reconstruction in the region $7 \mathrm{deg}<\theta<30 \mathrm{deg}$. The tracking system is surrounded by a finely segmented liquid argon (LAr) calorimeter, which covers the polar angle range $4 \mathrm{deg}<\theta<154 \mathrm{deg}$ with full azimuthal acceptance. The backward region ( $153 \mathrm{deg}<\theta<177.8 \mathrm{deg}$ ) is covered by a lead/scintillating-fibre calorimeter called the SpaCal; its main purpose is the detection of scattered positrons.

Neutral particles produced at very small polar angles with respect to the proton beam direction can be detected in the FNC, which is situated at a polar angle of $0 \mathrm{deg}$ at $106 \mathrm{~m}$ from the interaction point. A schematic view of the FNC is shown in Fig. 2 and the detailed description is given in $[7,8]$. The FNC is a lead-scintillator sandwich calorimeter. It consists of two longitudinal sections: the Preshower Calorimeter with a length corresponding to about 60 radiation lengths or $1.6 \lambda$ and the Main Calorimeter with a total length of $8.9 \lambda$ The acceptance of the FNC is defined by the aperture of the HERA beamline magnets and is limited to scattering angles of $\theta \lesssim$ $0.8 \mathrm{mrad}$ with approximately $30 \%$ azimuthal coverage (see Fig. 2).

The longitudinal segmentation of the FNC allows efficient discrimination of photons from neutrons. Photons are absorbed completely in the Preshower Calorimeter, while neutrons have a significant fraction of their energy deposited in the Main Calorimeter. Therefore, energy deposits in the FNC, which are contained in the Preshower Calorimeter with no energy deposits above the noise level in the Main Calorimeter, are classified as electromagnetic clusters. According to the Monte Carlo simulation about $98 \%$ of all reconstructed photon and neutron candidates originate from generated photons and neutrons, respectively. Due to the relatively large size of the FNC readout modules in combination with the small geometrical acceptance window, two or more particles entering the FNC are reconstructed as a single cluster. At lower energies the electromagnetic clusters reconstructed in the FNC mainly originate from single photons. At higher measured energies there is a significant

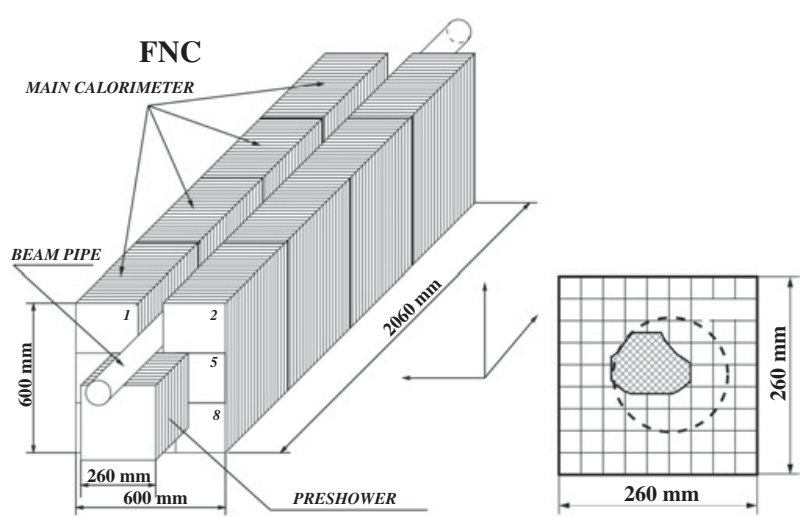

Figure 2. Left: a schematic view of the H1 FNC. Right: layout of the Preshower Calorimeter. The hatched area shows the geometrical acceptance window defined by the beam-line elements. The area corresponding to $\eta>7.9$ is indicated by dashed circle.

contribution from two photons, with the fraction of twophoton events increasing from $0.5 \%$ at $100 \mathrm{GeV}$ to $10 \%$ at about $450 \mathrm{GeV}$ and to $80 \%$ at $900 \mathrm{GeV}$. The two photons typically originate from the decay of the same meson.

The absolute electromagnetic and hadronic energy scales of the FNC are known to 5\% [8] and $2 \%$ [7] precision, respectively. The energy resolution of the FNC calorimeter for electromagnetic showers is $\sigma(E) / E \approx 20 \% / \sqrt{E[\mathrm{GeV}]} \oplus 2 \%$ and for hadronic showers $\sigma(E) / E \approx 63 \% / \sqrt{E[\mathrm{GeV}]} \oplus 3 \%$, as determined in test beam measurements. The spatial resolution is $\sigma(x, y) \approx 10 \mathrm{~cm} / \sqrt{\mathrm{E}[\mathrm{GeV}]} \oplus 0.6 \mathrm{~cm}$ for hadronic showers starting in the Main Calorimeter. A better spatial resolution of about $2 \mathrm{~mm}$ is achieved for the electromagnetic showers and for those hadronic showers which start in the Preshower Calorimeter.

\subsection{Cross section definition}

The kinematics of semi-inclusive forward photon and neutron production are shown in Fig. 1a. This measurement is restricted to the DIS kinematic range, determined by the photon virtuality $6<Q^{2}<100 \mathrm{GeV}^{2}$ and inelasticity $0.05<y<0.6$. They are defined as

$$
Q^{2}=-q^{2}, \quad y=\frac{p \cdot q}{p \cdot k},
$$

where $p, k$ and $q$ are the four-momenta of the incident proton, the incident positron and the virtual photon, respectively. The $\mathrm{CM}$ energy of the virtual photon-proton system, $W$, is related to $Q^{2}$ and $y$ as $W \approx \sqrt{y s-Q^{2}}$, where $s$ is the squared total CM energy of the positronproton system. The present analysis is restricted to the range $70<W<245 \mathrm{GeV}$.

The analysis is performed in the pseudorapidity range $\eta>7.9$ for forward neutrons and photons. The pseudorapidity range $\eta>7.9$ corresponds to polar angles $\theta<0.75$ mrad. In the virtual photon-proton $\mathrm{CM}$ frame the neutron transverse momentum $p_{T}^{*}$ and the neutron $x_{F}$ are restricted to the range $0<p_{T}^{*}<0.6 \mathrm{GeV}$ and $0.1<x_{F}<$ 0.94 , respectively. For the forward photons measurement 
Table 1. Definition of the kinematic phase space of the measurements.

\begin{tabular}{|c|c|}
\hline \multicolumn{2}{|c|}{ NC DIS Selection } \\
\hline \multicolumn{2}{|c|}{$\begin{array}{c}6<Q^{2}<100 \mathrm{GeV}^{2} \\
0.05<y<0.6 \\
70<W<245 \mathrm{GeV}\end{array}$} \\
\hline Forward photons & Forward neutrons \\
\hline$\eta>7.9$ & $\eta>7.9$ \\
\hline $0.1<x_{F}<0.7$ & $0.1<x_{F}<0.94$ \\
\hline $0<p_{T}^{*}<0.4 \mathrm{GeV}$ & $0<p_{T}^{*}<0.6 \mathrm{GeV}$ \\
\hline \multicolumn{2}{|c|}{$\begin{array}{c}W \text { ranges for cross sections } \frac{1}{\sigma_{D I S}} \frac{d \sigma}{d x_{F}} \\
70<W<130 \mathrm{GeV} \\
130<W<190 \mathrm{GeV} \\
190<W<245 \mathrm{GeV}\end{array}$} \\
\hline
\end{tabular}

$p_{T}^{*}$ and $x_{F}$ are defined for the most energetic photon in the pseudorapidity range $\eta>7.9$ and are restricted to the range $0<p_{T}^{*}<0.4 \mathrm{GeV}$ and $0.1<x_{F}<0.7$. The requirement that $x_{F}$ is below 0.7 for photons ensures that the electromagnetic clusters reconstructed in the FNC mainly originate from single photons.

The kinematic phase space of the measurements is summarised in Table 1. Cross sections of neutrons and photons produced in the forward direction, normalised to the inclusive DIS cross section, $1 / \sigma_{D I S} d \sigma / d x_{F}$, are determined differentially in $x_{F}$ in three ranges of $W$. In addition, the cross section ratios integrated over $x_{F}$, $\sigma_{D I S}^{\gamma, n} / \sigma_{D I S}$, are measured as a function of $W$.

\subsection{Event selection}

The data sample of this analysis was collected using triggers which require the scattered positron to be measured in the SpaCal. The trigger efficiency is about $96 \%$ for the analysis phase space. The selection of DIS events is based on the identification of the scattered positron as the most energetic, isolated compact calorimetric deposit in the SpaCal. A subsample of events containing forward photons or neutrons is selected by requiring either an electromagnetic or a hadronic cluster in the FNC with a pseudorapidity above 7.9 and an energy above $92 \mathrm{GeV}$. The DIS data sample contains about 9.3 million events. The FNC data sample contains about 83,000 events with photons and 230,000 events with neutrons.

\subsection{Monte Carlo simulations and corrections to the data}

Monte Carlo simulations are used to correct the data for the effects of detector acceptance, inefficiencies, QED radiation from the positron and migrations between measurement bins due to the finite detector resolution.

The DJANGOH [19] program is used to generate inclusive DIS events. It is based on leading order electroweak cross sections and takes into account QCD effects up to order $\alpha_{s}$. Higher order QCD effects are simulated using leading log parton showers as implemented in LEPTO [20], or using the Colour Dipole Model (CDM) as implemented in ARIADNE [21]. Subsequent hadronisation effects are modelled using the Lund string fragmentation model as implemented in JETSET $[22,23]$. Higher order electroweak processes are simulated using an interface to HERACLES [24]. In all DJANGOH simulations forward particles originate exclusively from the hadronisation of the proton remnant and forward photons are therefore mainly produced from the decay of $\pi^{0}$ mesons.

RAPGAP [25] is a general purpose event generator for inclusive and diffractive $e p$ interactions. In the version denoted below as RAPGAP- $\pi$, the program simulates exclusively the scattering of virtual photons off an exchanged pion (Fig. 1b). The parameterisations of the pion flux is taken from [26]. Using other parameterisations of pion flux affects mainly the absolute normalisation by up to $30 \%$. The best description of the forward neutron data is achieved by a combination of events with neutrons originating from pion exchange, as simulated by RAPGAP- $\pi$, and events with neutrons from proton remnant fragmentation, simulated by DJANGOH. RAPGAP- $\pi$ mainly contributes at high neutron energies, while DJANGOH is significant at low energies.

The measurements are also compared to predictions of several hadronic interaction models which are commonly used for the simulation of cosmic ray air shower cascades: EPOS LHC [27,28], QGSJET 01 [29,30], QGSJET II$04[31,32]$ and SIBYLL $2.1[33,34]$. The programs are interfaced with the PHOJET program [35] for the generation of the $e p$ scattering kinematics.

The measured distributions may contain background arising from several sources. The background from photoproduction processes is estimated using the PHOJET MC generator and is found to be about $1 \%$ on average. Background from misidentification of photons or neutrons in the FNC is estimated from the DJANGOH MC simulation to be $2 \%$ on average. These two backgrounds are subtracted from the data distributions bin-by-bin. Background also arises from a random coincidence of DIS events, causing activity in the central detector, with a beam-related background signal in the FNC, produced from the interaction of another beam proton with a positron or with residual gas in the beampipe. It is found to be smaller than $1 \%$ and is neglected.

The MC simulations are used to correct the distributions at the level of reconstructed particles back to the hadron level on a bin-by-bin basis. The correction factors are determined from the MC simulations as the ratios of the normalised cross sections obtained from particles at hadron level without QED radiation to the normalised cross sections calculated using reconstructed particles and including QED radiation effects. For the forward photon analysis the average of the correction factors determined from LEPTO and CDM is used. For the forward neutron analysis the correction factors are calculated using the combination of RAPGAP- $\pi$ and CDM simulations, with the weighting factors 0.6 and 1.4 , as described above. 


\section{Results}

\subsection{Normalised cross sections as a function of $W$}

The ratios of the forward photon and forward neutron production cross sections to the inclusive DIS cross section, $\frac{\sigma_{\text {DIS }}^{\gamma, \mathrm{n}}}{\sigma_{\text {DIS }}}$, in the kinematic range $6<Q^{2}<100 \mathrm{GeV}^{2}$ and $0.05<y<0.6$ and as a function of $W$ are shown in Fig. 3. Within uncertainties the measured ratios are consistent with constant values of about 0.027 (forwards photons) and 0.083 (forward neutrons). In other words, within uncertainties the $W$ dependence of the cross section is independent of the presence of a forward neutron or a forward photon, as predicted by the limiting fragmentation hypothesis $[10,11]$.

In Fig. 3 the MC model calculations are compared with the measurements. Both CDM and LEPTO predict a forward photon rate of about $70 \%$ higher than observed. The photon production rate as a function of $W$ is rather flat in CDM and shows a slight increase with $W$ in LEPTO. The shape of the $W$ distribution is in both models consistent with the data, within errors.

The rate of forward neutron production predicted by LEPTO is consistent with the data, while CDM predicts a much lower rate. However, as was shown in the previous measurement [7], the energy distribution of forward neutrons can be described by MC simulation only if this includes contributions both from standard fragmentation as simulated in DJANGOH, and from a pion exchange mechanism as explicitly simulated in RAPGAP- $\pi$ but not included in DJANGOH. In Fig. $3 b$ the combinations of the RAPGAP- $\pi$ and DJANGOH simulations, as described in Sect. 2.4, are compared to the measurement. The weighting factors 1.4 for the CDM, 0.7 for the LEPTO and 0.6 for the RAPGAP- $\pi$ predictions are determined by fitting the observed neutron energy distributions integrated over the full $W$ range. The cross sections for inclusive DIS events, used for the normalisation of the forward neutron cross sections, $\sigma_{\text {DIS }}$, are taken from the CDM and LEPTO simulations without additional weighting. The model combination describes the observed $W$ dependence well. It is remarkable that the factors for the CDM and LEPTO contributions differ by a factor two (1.4 and 0.7 , respectively). It is also notable that the CDM model, which overestimates the rate of forward photons by about $70 \%$, has to be scaled up in the combination to describe the forward neutron data.

In Fig. 3 predictions of various cosmic ray hadronic interaction models (EPOS LHC, SIBYLL 2.1 and the two versions of QGSJET) are compared to the measured normalised cross sections as a function of $W$. The CR model predictions show significant differences in absolute values, for both forward photons and forward neutrons. For photons all models predict too high rates by 30 to $40 \%$, and these rates, with the exception of EPOS LHC, show a slight decrease with increasing $W$, not confirmed by data. For forward neutrons all CR predictions show a $W$ independent behaviour, in accordance with the measured $W$ dependence. The QGSJET 01 model predicts a much too high and SIBYLL 2.1 a much too low neutron rate,
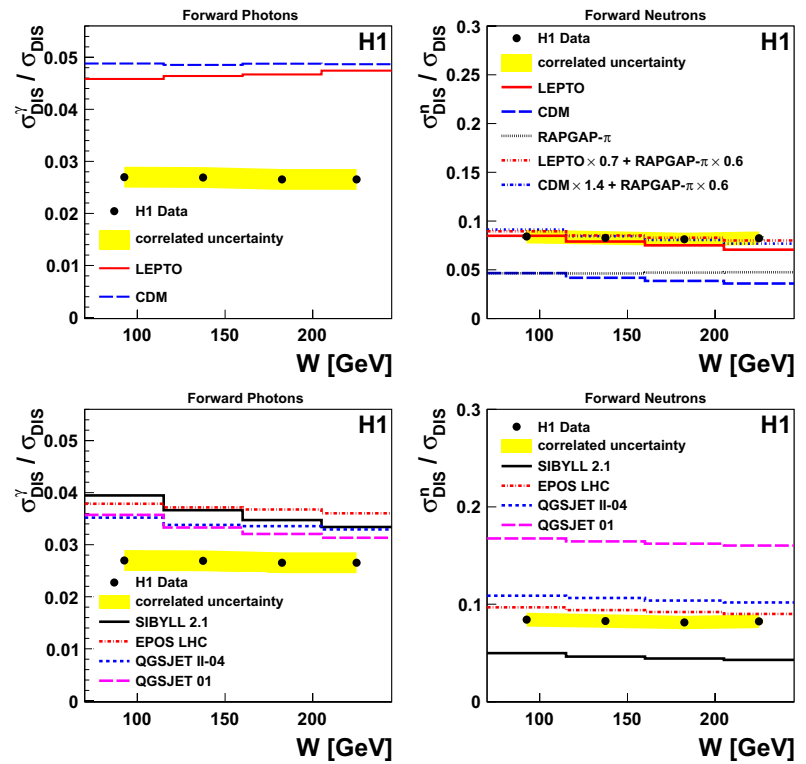

Figure 3. The fraction of DIS events with forward photons (left side plots) and forward neutrons (right side plots) as a function of $W$. In the upper plots also shown are the predictions of the LEPTO and CDM and, in the case of forward neutron production, the predictions of RAPGAP- $\pi$ and the linear combinations of LEPTO and RAPGAP- $\pi$, as well as CDM and RAPGAP- $\pi$. In the lower plots also shown are the predictions of the CR hadronic interaction models SIBYLL 2.1, QGSJET 01, QGSJET II-04 and EPOS LHC.

while the EPOS LHC and QGSJET II-04 models are closer to the measurement.

\subsection{Normalised cross sections as a function of $x_{F}$ and test of Feynman scaling}

The measured normalised differential cross sections, $\frac{1}{\sigma_{\text {DIS }}} \frac{\mathrm{d} \sigma}{\mathrm{dx}_{\mathrm{F}}}$, of the most energetic photon are presented as a function of $x_{F}$ in Figs. 4 and 5 for the kinematic region defined in Table 1. In order to study the energy dependence of the $x_{F}$ distributions, these cross sections are measured in three $W$ intervals.

The normalised differential cross sections as a function of $x_{F}$ are similar for the three $W$ ranges. As shown in Fig. 4 and already seen in the comparison of the $W$ dependence, the LEPTO and CDM models predict a rate of forward photons about $70 \%$ higher than measured. The shapes of the measured distributions are well described by LEPTO, while the CDM description is very poor by showing a significantly harder spectrum than observed in data.

In Fig. 5 the predictions of the CR hadronic interaction models are compared to the same measurements. Large differences between the CR models are observed, both in shape and in normalisation. All models tested here overestimate the forward photon rate by $30 \%$ to $40 \%$ at low $x_{F}$. The EPOS LHC model describes the shapes of the photon $x_{F}$ distributions well. The SIBYLL 2.1 model predicts a harder $x_{F}$ dependence, while the spectra obtained from the different variants of QGSJET are softer than observed in the data. 

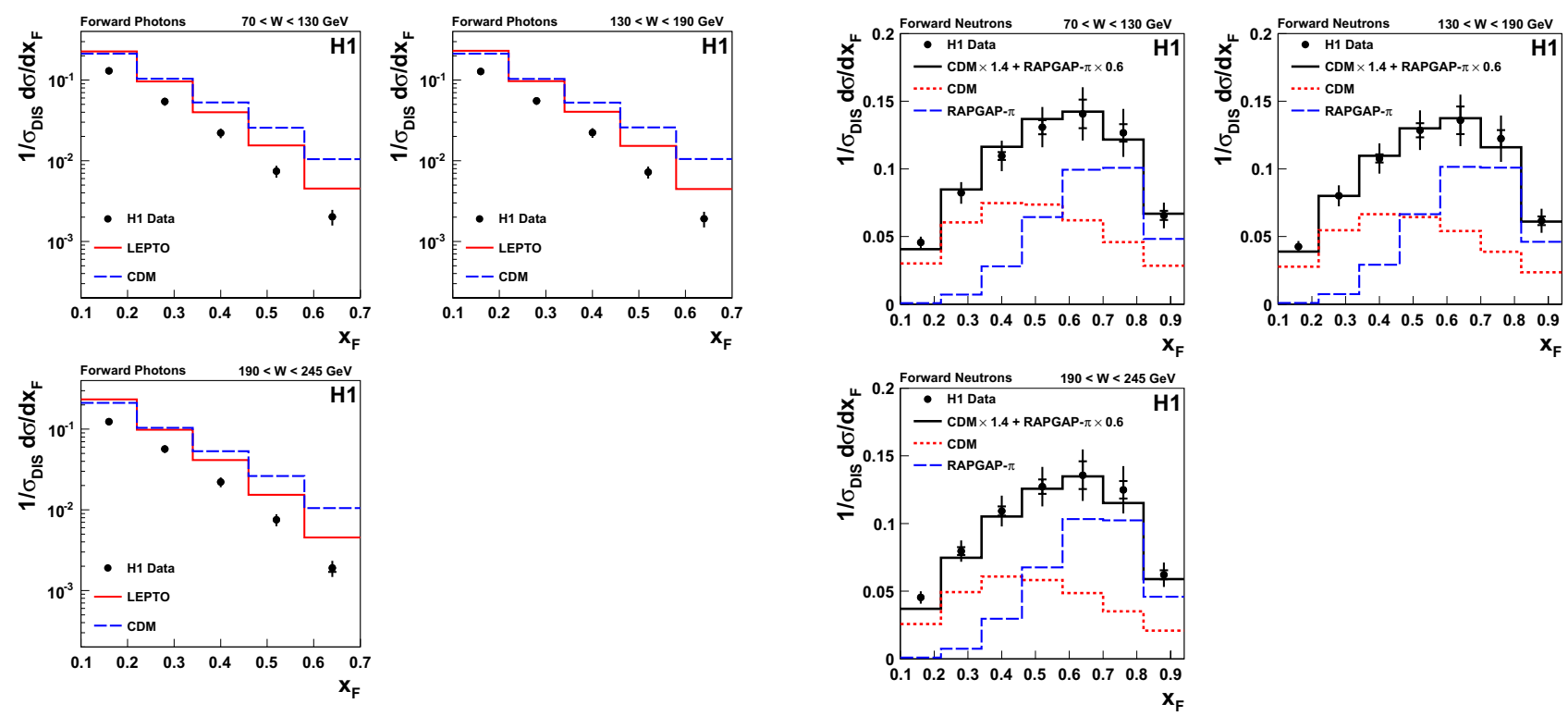

Figure 4. Normalised cross sections of forward photon production in DIS as a function of $x_{F}$ in three $W$ intervals in the kinematic region given in Table 1. Also shown are the predictions of the LEPTO and CDM MC models.
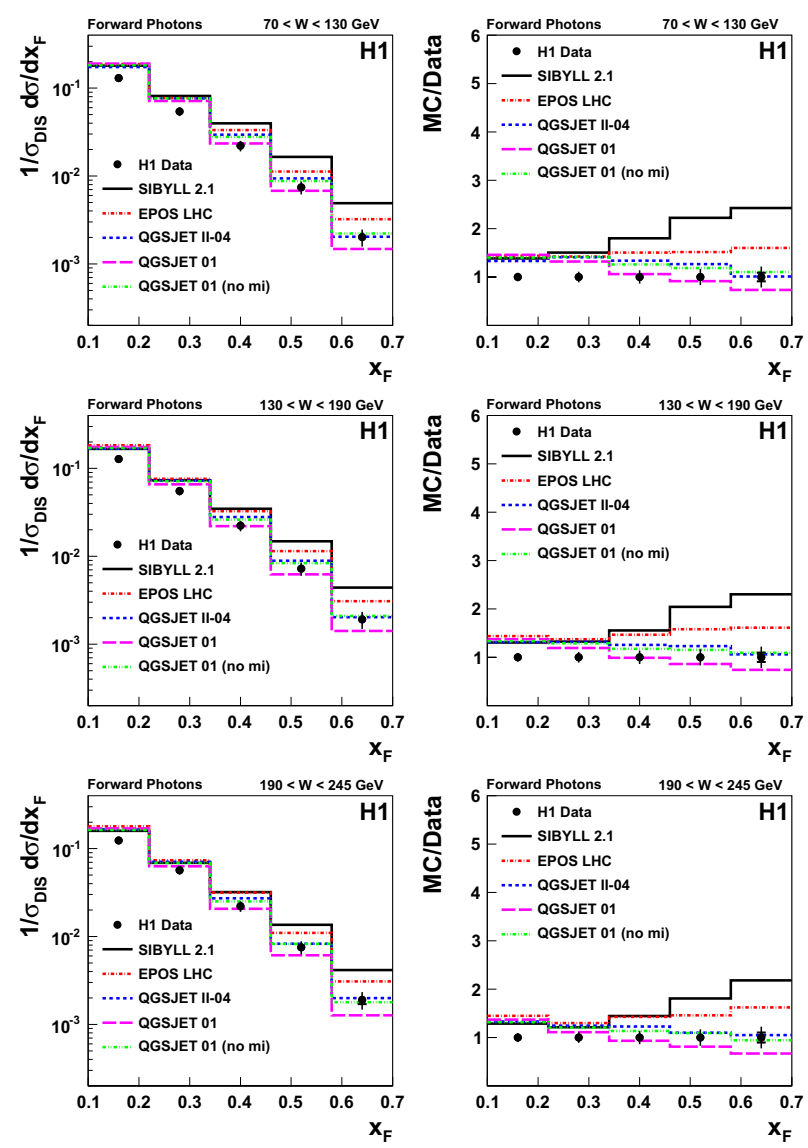

Figure 5. Normalised cross sections of forward photon production in DIS as a function of $x_{F}$ in three $W$ intervals in the kinematic region given in Table 1. Also shown are the predictions of the cosmic ray hadronic interaction models SIBYLL 2.1, QGSJET 01, QGSJET 01 (no mi), QGSJET II04 and EPOS LHC. In the right column the ratio of CR model predictions to the data are shown.

Figure 6. Normalised cross sections of forward neutron production in DIS as a function of $x_{F}$ in three $W$ intervals in the kinematic region given in Table 1. Also shown are the predictions of CDM, RAPGAP- $\pi$ and the linear combination of CDM and RAPGAP- $\pi$ predictions.

The normalised differential cross sections for the forward neutron are presented in in Figs. 6 and 7 for the kinematic region defined in Table 1 . The $x_{F}$ distributions of forward neutrons are well described by a combination of CDM and RAPGAP- $\pi$, using the weighting factors and normalisation as described in Sect. 3.1. The individual contributions of the two models are shown in Fig. 6 as well. Fragmentation, as simulated by CDM, dominates the neutron production at lower $x_{F}$, while the contribution from pion exchange becomes significant at $x_{F} \gtrsim 0.7$. The combination of LEPTO and RAPGAP- $\pi$ (not shown) also provides a good description of the measurements for all $W$ ranges. In Fig. 7 the predictions of the CR hadronic interaction models are compared to the forward neutron production cross sections. The EPOS LHC model provides a reasonable description of the neutron $x_{F}$ distributions, except at the highest $x_{F}$ values. The SIBYLL 2.1 model describes the shape of the $x_{F}$ spectra but fails in the absolute rate. The QGSJET II-04 model shows a harder $x_{F}$ dependence, and QGSJET 01 predicts a much too high neutron rate.

It was pointed out [36] that the hadronic interaction models have been developed for hadron-hadron interactions and therefore the simulation of DIS events might be affected by the superfluous contribution of multi-parton interactions. In order to investigate this assumption, the QGSJET 01 model has been modified [36] to exclude the multi-parton interactions. In the comparison with the measurements this modified model is denoted as "QGSJET 01 (no mi)". As seen in Figs. 5 and 7 the effect of multi-parton interactions on the forward photon production is small, while the $x_{F}$ spectrum of forward neutrons becomes significantly harder, when multi-parton interactions are excluded, without improving the data description. 

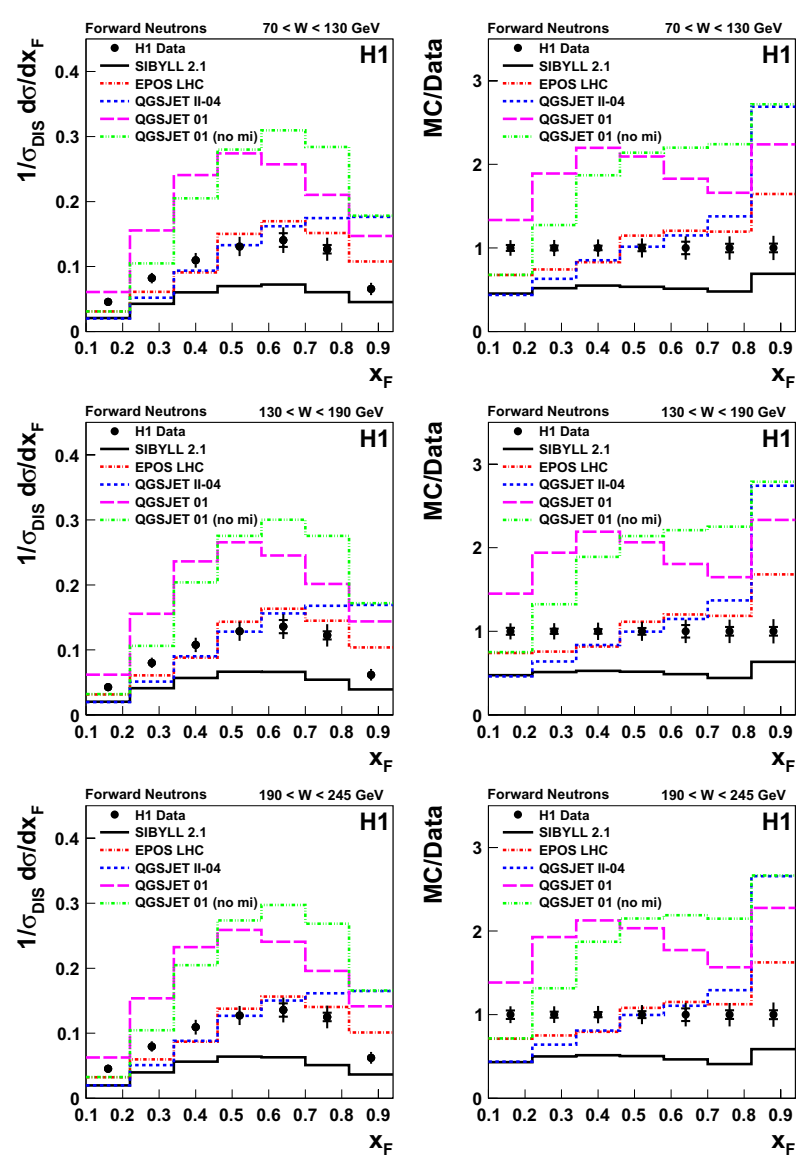

Figure 7. Normalised cross sections of forward neutron production in DIS as a function of $x_{F}$ in three $W$ intervals in the kinematic region given in Table 1. Also shown are the predictions of the CR hadronic interaction models SIBYLL 2.1, QGSJET 01, QGSJET 01 (no mi), QGSJET II-04 and EPOS LHC. In the right column the ratio of $\mathrm{CR}$ model predictions to the data are shown.

The $W$ dependence of the $x_{F}$ distributions allows a test of the Feynman scaling hypothesis for particle production. For this test, the ratios of the normalised cross sections for different $\mathrm{CM}$ energy intervals are studied as a function of $x_{F}$. Figure 8 show the ratios of the second to the first and the third to the first $W$ range for photons. In Fig. 9 the same ratios are shown for forward neutrons. For all data distributions the values of these ratios are roughly consistent with unity and being constant within uncertainties, suggesting that Feynman scaling in the target fragmentation region holds for photons and neutrons. All CR models indicate deviations from scaling for the forward photons, such that the production rate decreases with increasing $W$. In particular, this effect is strong for SIBYLL 2.1 and QGSJET 01 models. For forward neutrons the CR models are consistent with Feynman scaling, with exception of SIBYLL 2.1.

\section{Summary}

The production of high energy forward neutrons and photons has been studied at HERA in deep-inelastic ep scattering in the kinematic region $6<Q^{2}<100 \mathrm{GeV}^{2}$ and $0.05<y<0.6$. The normalised DIS cross sections
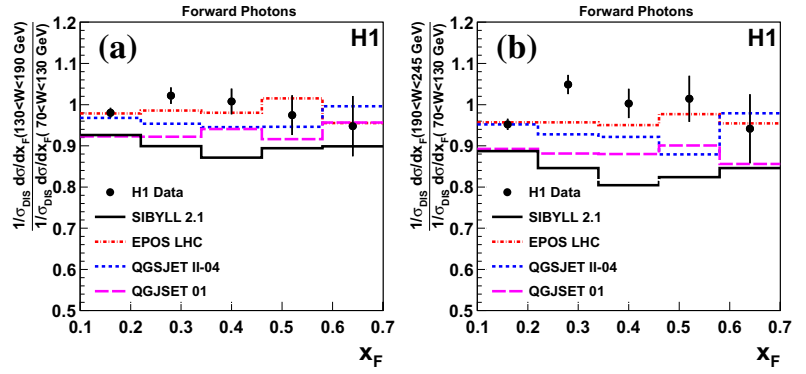

Figure 8. Ratios of normalised cross sections of forward photon production in DIS corresponding to two different $W$ intervals, shown in Fig. 5, as a function of $x_{F}$ : (a) ratio of the cross section in the $130<W<190 \mathrm{GeV}$ interval to the cross section in the $70<W<130 \mathrm{GeV}$ interval; (b) ratio of the cross section in the $190<W<245 \mathrm{GeV}$ interval to the cross section in the $70<W<130 \mathrm{GeV}$ interval. Also shown are the predictions of the CR hadronic interaction models SIBYLL 2.1, QGSJET 01, QGSJET II-04 and EPOS LHC.
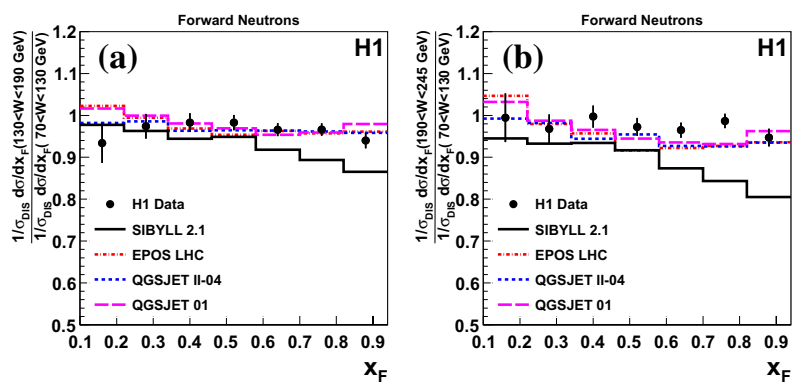

Figure 9. Ratios of normalised cross sections of forward neutron production in DIS corresponding to two different $W$ intervals, shown in Fig. 7, as a function of $x_{F}$ : (a) ratio of the cross section in the $130<W<190 \mathrm{GeV}$ interval to the cross section in the $70<W<130 \mathrm{GeV}$ interval; (b) ratio of the cross section in the $190<W<245 \mathrm{GeV}$ interval to the cross section in the $70<W<130 \mathrm{GeV}$ interval. Also shown are the predictions of the CR hadronic interaction models SIBYLL 2.1, QGSJET 01, QGSJET II-04 and EPOS LHC.

$1 / \sigma_{D I S} d \sigma / d x_{F}$ for the production of photons and neutrons at pseudorapidities $\eta>7.9$ and in the range of Feynman- $x$ of $0.1<x_{F}<0.7$ for the photons and $0.1<$ $x_{F}<0.94$ for neutrons are presented. The measured cross sections as a function of $x_{F}$ at different centre-of-mass energies of the virtual photon-proton system agree within uncertainties, confirming the validity of Feynman scaling in the energy range of the virtual photon-proton system $70<W<245 \mathrm{GeV}$.

Different Monte Carlo models are compared to the measurements. All these models overestimate the rate of photons by $30-70 \%$. The shapes of the measured forward photon cross sections are well described by the LEPTO MC simulation, while predictions based on the colour dipole model fail, especially at high $x_{F}$. The cross sections for forward neutrons are well described by a linear combination of the standard fragmentation model, as implemented in DJANGOH, and the onepion-exchange model RAPGAP- $\pi$. Predictions of models, which are commonly used for the simulation of cosmic ray cascades, are also compared to the forward photon 
and neutron measurements. None of the models describes the photon and neutron data simultaneously well. The best description of the shapes of the photon and the neutron $x_{F}$ distributions is provided by the EPOS LHC model. Within the kinematic range of the measurements, the relative rate of forward photons and neutrons in DIS events is observed to be independent of the energy of the virtual photon-proton $\mathrm{CM}$, and therefore also consistent with the hypothesis of limiting fragmentation. The present measurement provides new information to further improve the understanding of proton fragmentation in collider and cosmic ray experiments.

I am grateful to the Organisers for giving me the possibility to attend the Symposium and present this talk.

\section{References}

[1] R. Engel, Nucl. Phys. Proc. Suppl. 75A (1999) 62 [astro-ph/9811225]

[2] A. Buniatyan et al., "Cosmic Rays, HERA and the LHC: Working group summary.” Proceedings of the Workshop on the Implications of HERA for LHC Physics, Geneva, Switzerland, 26-30 May 2008, p.566, DESY-PROC-2009-02 [arXiv:0903.3861]

[3] C. Adloff et al. [H1 Collaboration], Eur. Phys. J. C6 (1999) 587 [hep-ex/9811013]

[4] S. Chekanov et al. [ZEUS Collaboration], Nucl. Phys. B637 3 (2002) 3 [hep-ex/0205076]

[5] S. Chekanov et al. [ZEUS Collaboration], Nucl. Phys. B776 (2007) 1 [hep-ex/0702028]

[6] S. Chekanov et al. [ZEUS Collaboration], JHEP 06 (2009) 074 [arXiv:0812.2416]

[7] F. Aaron et al. [H1 Collaboration], Eur. Phys. J. C68 (2010) 381 [arXiv:1001.0532]

[8] F. Aaron et al. [H1 Collaboration], Eur. Phys. J. C71 (2011) 1771 [arXiv:1106.5944]

[9] V. Andreev et al. [H1 Collaboration], Eur. Phys. J. C 74, 2915 (2014) [arXiv:1404.0201]

[10] J. Benecke et al., Phys. Rev. 188 (1969) 2159.

[11] T. T. Chou and C.-N. Yang, Phys. Rev. D50 (1994) 590

[12] R. P. Feynman, Phys. Rev. Lett. 23 (1969) 1415.
[13] E. Pare et al. [UA7 Collaboration], Phys. Lett. B242 (1990) 531

[14] A. Ohsawa, Prog. Theor. Phys. 92 (1994) 1005

[15] I. Abt et al. [H1 Collaboration], Nucl. Instrum. Meth. A386 (1997) 310

[16] I. Abt et al. [H1 Collaboration], Nucl. Instrum. Meth. A386 (1997) 348

[17] R. D. Appuhn et al. [H1 SPACAL Group], Nucl. Instrum. Meth. A386 (1997) 397

[18] D. Pitzl et al., Nucl. Instrum. Meth. A454 (2000) 334 [hep-ex/0002044]

[19] K. Charchula, G. A. Schuler and H. Spiesberger, DJANGOH 1.4, Comput. Phys. Commun. 81 (1994) 381

[20] G. Ingelman, A. Edin and J. Rathsman, LEPTO 6.5, Comput. Phys. Commun. 101 (1997) 108 [hep-ph/9605286]

[21] L. Lönnblad, ARIADNE 4.10, Comput. Phys. Commun. 71 (1992) 15

[22] B. Andersson et al., Phys. Rep. 97 (1983) 31

[23] T. Sjöstrand, PYTHIA 5.7 and JETSET 7.4, [hep-ph/9508391]

[24] A. Kwiatkowski, H. Spiesberger and H. J. Möhring, Comp. Phys. Commun. 69 (1992) 155

[25] H. Jung, RAPGAP 3.1, Comp. Phys. Commun. 86 (1995) 147

[26] H. Holtmann et al., Phys. Lett. B338 (1994) 363

[27] K. Werner, F.-M. Liu and T. Pierog, Phys. Rev. C74 (2006) 044902 [hep-ph/0506232]

[28] T. Pierog et al., DESY-13-125 [arXiv: 1306.0121]

[29] N. N. Kalmykov and S. S. Ostapchenko, Phys. Atom. Nucl. 56 (1993) 346

[30] N. N. Kalmykov, S. S. Ostapchenko and A. I. Pavlov, Nucl. Phys. Proc. Suppl. 52B (1997) 17

[31] S. S. Ostapchenko, Phys. Rev. D74 (2006) 014026 [hep-ph/0505259]

[32] S. S. Ostapchenko, Phys. Rev. D83 (2011) 014018 [arXiv:1010 . 1869]

[33] J. Engel et al., Phys. Rev. D46 (1992) 5013

[34] E.-J. Ahn et al., Phys. Rev. D80 (2009) 094003 [arXiv:0906.4113]

[35] R. Engel and J. Ranft, Phys. Rev. D54 (1996) 4244 [hep-ph/9509373]

[36] S. S. Ostapchenko and T. Pierog, Private communication 